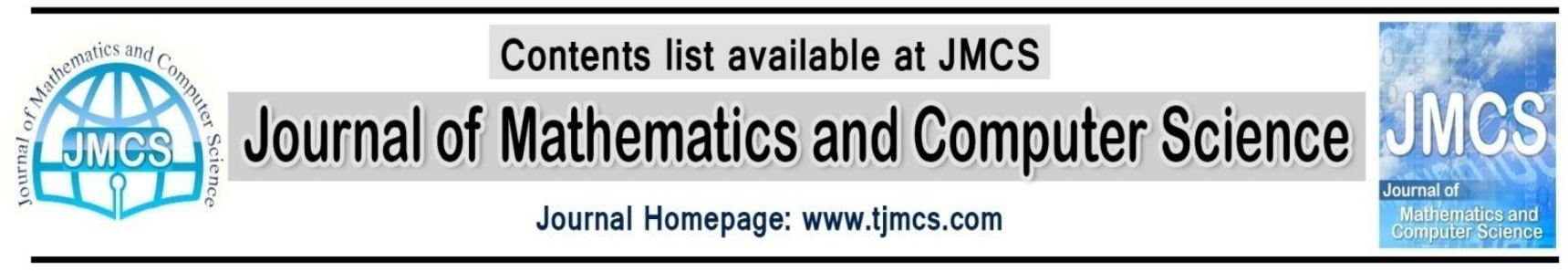

\title{
Further Results on Harmonic Index and Some New Relations Between Harmonic Index and Other Topological Indices
}

\author{
Khosro Sayehvand ${ }^{\text {a, }}{ }^{*}$, Mohammadreza Rostami ${ }^{\text {a }}$ \\ ${ }^{a}$ Faculty of Mathematical Sciences, University of Malayer, P. O. Box 16846-13114, Malayer, Iran \\ ksayehvand@malayeru.ac.ir, rostami.mohamadreza@gmail.com
}

Article history:

Received April 2014

Accepted May 2014

Available online June 2014

Abstract
The harmonic index $H(G)$ of a graph $G$ is defined as the sum of the weights $\frac{2}{d_{u}+d_{v}}$ of all edges $u v$ of $G$, where $d_{u}$ denotes the degree of a vertex $u$ in $G$. In this paper, we obtained some new relationships between harmonic index and first geometric-arithmetic index, sum connectivity index that this indices are important than another topological index. In addition, we determine the lower and upper bond for molecular graphs and unicyclic molecular graph. Also we give a characterization of the minimum harmonic index of graphs with maximum degree $\Delta$.

\section{Mathematics Subject Classification: 05 C90.}

Keywords: harmonic index, first geometric-arithmetic index, sum connectivity index, molecular graph, minimum degree, maximum degree.

\section{Introduction}

Let $G=(V, E)$ be a graph with vertex sets $V(G)$ and edge sets $E(G)$. Also let $|V(G)|=n$ and $|\boldsymbol{E}(\boldsymbol{G})|=\boldsymbol{m}$. The harmonic index of graph $\mathrm{G}$ is defined as:

$$
H(G)=\sum_{u v \in(G)} \frac{2}{d_{u}+d_{v}}
$$

where $d_{u}$ is the degree of a vertex $u$ in $G$. 
As far as we know, this index first appeared in [1]. Favaron et al. [2] considered the relation between the harmonic index and the eigenvalues of graphs. Here we will consider the minimum and maximum values of the harmonic index for simple connected graphs and trees, and characterize the corresponding extremal graphs. Zhong in [3] presented the minimum andmaximum values of harmonic index on simple connected graphs and trees, and characterized the corresponding extremal graphs. Also, Zhong [3], obtained the unicyclic graphs with the minimum and maximum harmonic index among all $n$-vertex unicyclic graphs. Wua et al. [4] obtain the lower bound for the harmonic index of a graph with minimum degree at least two and characterize the extremal graphs. For the more study in harmonic index may be found in $[5,6,7$, 8].

The topological index that is named as first geometric-arithmetic index $\left(G A_{1}\right)$ is defined as [9]:

$$
G A_{1}(G)=\sum_{u v \in E(G)} \frac{2 \sqrt{d_{u} d_{v}}}{d_{u}+d_{v}}
$$

It found applications of first geometric arithmetic index in chemical research in this paper [9].Upper and lower bounds for the first geometric-arithmetic index of general graphs and molecular graph and molecular tree have been given in $[9,10]$.

Sum connectivity index is the one another topological index those descriptor truly molecular properties. This index is defined as

$$
\chi(G)=\sum_{u v \in E(G)} \frac{1}{\sqrt{d_{u}+d_{v}}} .
$$

In [11], the authors provided several basic properties for sum-connectivity index, especially lower and upper bounds in terms of sum-connectivity index, and they determined a unique tree with the given numbers of vertices and pendant vertices with the minimum value of the sumconnectivity index, and trees with the minimum, second minimum and third minimum, and with the maximum, second maximum and third maximum values of the sum-connectivity index. In [12], some properties of the sum-connectivity index were obtained for trees and unicycle graphs with given matching number. The most important works on Sum-connectivity index of molecular trees were done by Xing and his colleagues [13].

Recently, many researcher works on harmonic index. In [4] the authors obtained lower bound for the harmonic index of a graph with minimum degree at least two. Deng and et al. have studied the relation between harmonic index and chromatic number of a graph in [6]. In the paper [5] author established some relationships between harmonic index and randic index and atom-bond connectivity index. In this paper we determine the relationship between harmonic index and first geometric arithmetic index and sum connectivity index that they are important topological indices.

In Section 2 we have introduced the basic of graph and definition, and notation on graph which applied to another sections. In Section 3 we obtain the relations between the harmonic index and first geometric-arithmetic index and sum connectivity index, for general graph and the special class of graphs. In Section 4 we obtain the lower and upper bond for molecular graphs and unicyclic molecular graphs. And we characterize the external molecular graph with the minimum degree is two. Also we give the lower bond of among all graphs with maximum degree $\Delta$. Finally in Section 5 we calculate the harmonic, geometric-arithmetic and sum connectivity 
indices for octane isomers and show that the harmonic index can be used in QSPR and QSAR research.

\section{Preliminaries}

Let $K_{n}, C_{n}, S_{n}$ and $P_{n}$ be the complete graph, cycle, star and path on $n$ vertices, respectively. Let $K_{n, m}$, be the complete bipartite graph on $n$ and $m$ vertices in its two partition sets. The complement $\bar{G}$ of a simple graph $G$ is the simple graph with vertex set $V$, two vertices being adjacent in $\bar{G}$ if and only if they are not adjacent in $G$ [14]. The maximum possible vertex degree in an obituary graph is $n-1$. Denote by $n_{i}$ the number of vertices of degree $i$ in $G$ and $x_{i, j}$ the number of edges joining the vertices of degrees $i$ and $j$ in graph $G$. It is clear that for obituary connected graph we have $x_{i, j}=x_{j, i}$ and $n_{0}=0$. Then the $H, G A_{1}$ and $\chi$ indices can be written as:

$$
\begin{aligned}
& H(G)=\sum_{1 \leq i \leq j \leq n-1} \frac{2}{i+j} x_{i, j}, \\
& G A_{1}(G)=\sum_{1 \leq i \leq j \leq n-1} \frac{2 \sqrt{i j}}{i+j} x_{i, j}, \\
& \chi(G)=\sum_{1 \leq i \leq j \leq n-1} \frac{1}{\sqrt{i+j}} x_{i, j} .
\end{aligned}
$$

\section{Relations between harmonic index and another topological indices}

In this section, we established some relationships between harmonic index and first geometric-arithmetic index and sum connectivity index, for general graph and the special class of graphs.

Corollary 1: Let $G$ be a connected graph with $n \geq 2$ vertices. It is clear that for any edge of graph $G$ we have, $1 \leq d_{u} d_{v}$. Consequently

$$
\frac{2}{d_{u}+d_{v}} \leq \frac{2 \sqrt{d_{u} d_{v}}}{d_{u}+d_{v}},
$$

then,

$$
H(G) \leq G A_{1}(G) .
$$

The equality occurs if and only if $d_{u}=d_{v}=1$ for any edge $u v \in E(G)$, which implies $G \cong K_{2}$. 
Corollary2: Let $G$ be a connected graph with $n \geq 2$ vertices. For any vertex $u$ of graph $G$, we have $d_{u} \leq n-1$, then $\sqrt{d_{u} d_{v}} \leq n-1$. So

$$
G A_{1}(G)=\sum_{u v E(G)} \frac{2 \sqrt{d_{u} d_{v}}}{d_{u}+d_{v}} \leq(n-1) \sum_{u v E(G)} \frac{2}{d_{u}+d_{v}}=(n-1) H(G) .
$$

then,

$$
(n-1) H(G) \geq G A_{1}(G) .
$$

The equality occurs if and only if

$$
d_{u}=d_{v}=n-1,
$$

for any edge $u v \in E(G)$, which implies $G \cong K_{n}$.

Corollary3: Let $S_{n}$ be the star tree with $n$ vertices, then,

$$
\left\{\begin{array}{lll}
H\left(S_{n}\right)>\chi\left(S_{n}\right) & \text { if } & n=2,3, \\
H\left(S_{n}\right)=\chi\left(S_{n}\right) & \text { if } & n=4, \\
H\left(S_{n}\right)<\chi\left(S_{n}\right) & \text { if } & n \geq 5 .
\end{array}\right.
$$

We know that for each edge $u v$ of $S_{n}$ have $d_{u}=1$ and $d_{v}=n-1$, then,

$$
H\left(S_{n}\right)=\sum_{u v E\left(S_{n}\right)} \frac{2}{d_{u}+d_{v}}=\frac{2(n-1)}{n},
$$

and

$$
\chi\left(S_{n}\right)=\sum_{u \in E\left(S_{n}\right)} \frac{1}{\sqrt{d_{u}+d_{v}}}=\frac{n-1}{\sqrt{n}} .
$$

So,

$$
H\left(S_{n}\right)-\chi\left(S_{n}\right)=\frac{2(n-1)}{n}-\frac{n-1}{\sqrt{n}}=\frac{n-1}{\sqrt{n}}\left(\frac{2}{\sqrt{n}}-1\right) .
$$

Then,

$$
H\left(S_{n}\right)<\chi\left(S_{n}\right) \Leftrightarrow\left(\frac{2}{\sqrt{n}}-1\right)<0 \Leftrightarrow n \geq 5 .
$$

By considering the above equality and numerical checking for $n=2,3,4$ we can conclude the following results.

Corollary 4: Let $G$ be a simple and connected graph with $n \geq 3$ vertices and there are not edge, that connect a vertex of degree one to the vertex of degree two $\left(x_{1,2}=0\right)$. For the harmonic index and sum connectivity index for any graph $G$ can be written as

and

$$
H(G)=\sum_{1 \leq i \leq j \leq n-1} \frac{2 x_{i, j}}{i+j},
$$




$$
\chi(G)=\sum_{1 \leq i \leq j \leq n-1} \frac{x_{i, j}}{\sqrt{i+j}}
$$

So,

$$
H(G)-\chi(G)=\sum_{1 \leq i \leq j \leq n-1}\left(\frac{2}{i+j}-\frac{1}{\sqrt{i+j}}\right) x_{i, j} .
$$

Now we consider the coefficient of $x_{i, j}$ in above series, then

$$
\frac{2}{i+j}-\frac{1}{\sqrt{i+j}} \leq 0 \Leftrightarrow-(i+j)(i+j-4) \leq 0 .
$$

Since $i+j \geq 4$, then above unequal is true and the equality occurs for $i=1, j=3$ and $i=j=2$. Consequently,

$$
H(G) \leq \chi(G) .
$$

With equality occurs only for $S_{4}$ and $C_{n}$.

Corollary 5: Let $G$ be a simple and connected graph with $n \geq 3$ vertices with $\delta \geq 2$. Then,

$$
H(G) \leq \chi(G)
$$

With equality occurs if and only if $G \cong C_{n}$.

Corollary6: Let $G$ be a simple and connected graph with $n \geq 3$ vertices and $d_{u}+d_{v} \leq 4$ for any $u v \in E(G)$. We have,

$$
H(G)-\chi(G)=\sum_{1 \leq i \leq j \leq n-1}\left(\frac{2}{i+j}-\frac{1}{\sqrt{i+j}}\right) x_{i, j} .
$$

Now we consider the coefficient of $x_{i, j}$ in above series, then

$$
\frac{2}{i+j}-\frac{1}{\sqrt{i+j}} \geq 0 \Leftrightarrow-(i+j)(i+j-4) \geq 0 .
$$

Then,

$$
H(G) \geq \chi(G)
$$

The equality occurs for $G \cong S_{4}$ or $G \cong C_{n}$. The above unequal is true for $i+j \leq 4$ and the equality occurs for $i=1, j=3$ and $i=j=2$.

Corollary 7: Let $P_{n}$ be the path with $n \geq 2$ vertices, path with $n \geq 2$ vertices has $n-1$ edges. By numerical calculations of harmonic index and sum connectivity index for $P_{2}$ we have, $1=H\left(P_{2}\right)>\chi\left(P_{2}\right)=\frac{\sqrt{2}}{2}$. Now we consider $n \geq 3$, then there are two edges that connect a vertex of degree one to the vertex of degree two ( on the other hand $x_{1,2}=2$ ) and other edges connect a vertex of degree two to the vertex of degree two ( on the other hand $x_{2,2}=n-3$ ), therefore by corollary 6 we have, $H\left(P_{n}\right)>\chi\left(P_{n}\right)$. 
Corollary 8: Let $G$ be a simple and connected $r$-regular graph with $n \geq 3$ vertices and $r \geq 2$. Then,

$$
H(G) \leq \chi(G) .
$$

The equality occurs if and only if $r=2$, which implies $G \cong C_{n}$.

Corollary 9: Let $G$ be a simple and connected graph with $n$ vertices and $m$ edges. It is clear that for any edge of graph $G$ we have $2 \leq d_{u}+d_{v} \leq 2 n-2$ then,

$$
\frac{1}{2 n-2} \leq \frac{1}{d_{u}+d_{v}} \leq \frac{1}{2}
$$

consequently

and

$$
\sum_{u \in E(G)} \frac{1}{n-1} \leq \sum_{u \in E(G)} \frac{2}{d_{u}+d_{v}} \leq \sum_{u v E(G)} 1
$$

$$
\frac{m}{n-1} \leq H(G) \leq m
$$

Furthermore, lower bound and upper bound are happens for $K_{n}$ and $K_{2}$.

Corollary 10: Let $G$ be a simple and connected graph on $n$ vertices with a connected complement $\bar{G}$. Let $m$ and $\bar{m}$ are the number of edge of $G$ and $\bar{G}$, respectively. Then by corollary 9 , we have

$$
\frac{\bar{m}}{n-1}+\frac{m}{n-1} \leq H(G)+H(\bar{G}) \leq \bar{m}+m,
$$

consequently

$$
\frac{n}{2} \leq H(G)+H(\bar{G}) \leq\left(\begin{array}{l}
n \\
2
\end{array}\right) .
$$

Corollary 11: Let $G$ be a simple and connected $r$-regular graph on $n \geq 3$ vertices and $r \geq 2$ with a connected complement $\bar{G}$. For any $r$-regular connected graph with $n \geq 3$ vertices and $r \geq 2$, we have $H(G) \leq \chi(G)$. It is clear that if $G$ be a $r$-regular connected graph with a connected complement $\bar{G}$, and then $\bar{G}$ is a $(n-r-1)$-regular connected graph. Then

$$
H(\bar{G}) \leq \chi(\bar{G}) .
$$

Above equality occurs if and only if $G \cong C_{n-r-1}$.

Corollary 12: Let $G$ be a simple and connected graph on $n$ vertices with a connected complement $\bar{G}$. Then

Upper bound does not happen.

$$
H(\bar{G})<G A_{1}(\bar{G}) .
$$

Corollary 13: Let $G$ be a complete bipartite graph with $n \geq 4$ vertices and $m$ edges. It is clear that for any $u v$ of complete bipartite graph $G$ we have 


$$
d_{u}+d_{v}=n,
$$

then,

$$
H(G)=\sum_{u v E(G)} \frac{2}{d_{u}+d_{v}}=\frac{2 m}{n} .
$$

So, the minimum and maximum value of $H(G)$ is depended of the edges of complete bipartite graph $G$. The complete bipartite graph $G$ that has the minimum value of edges is $K_{1, n}$ and the complete bipartite graph that has the maximum value of edges is $K_{\left\lfloor\frac{n}{2}\right\rfloor \mid\left\lceil\frac{n}{2}\right\rceil}$. Consequently, we will have

$$
\frac{2(n-1)}{n} \leq H(G) \leq \frac{2}{n}\left\lfloor\frac{n}{2}\right\rfloor\left\lceil\frac{n}{2}\right\rceil .
$$

Lower bound and upper bound are happens for $K_{1, n-1}$ and $K_{\left.\left\lfloor\frac{n}{2}\right] \mid \frac{n}{2}\right\rceil}$.

\section{Harmonic index of molecular graphs}

A connected graph with the maximum degree at most four is a molecular graph representing hydrocarbons [15]. Let $G$ be a molecular graph with $n \geq 3$ vertices and $m$ edges, then

$$
n-1 \leq m \leq 2 n .
$$

Theorem 14: Let $G$ be a molecular graph with $n \geq 3$ vertices and $m$ edges, then

$$
\frac{3 m+4 n}{20} \leq H(G) \leq \frac{m+2 n}{6} .
$$

With left equality if and only if $G$ has only vertices of degree one and four, and with right equality if and only if $G$ is either a path or a cycle.

Proof: We consider the notations on Section 2, so we can write

$$
\begin{aligned}
H(G)=\sum_{1 \leq i \leq j \leq 4} \frac{2}{i+j} x_{i, j}= & \frac{2}{3} x_{1,2}+\frac{1}{2} x_{1,3}+\frac{2}{5} x_{1,4}+\frac{1}{2} x_{2,2}+\frac{2}{5} x_{2,3} \\
& +\frac{1}{3} x_{2,4}+\frac{1}{3} x_{3,3}+\frac{2}{7} x_{3,4}+\frac{1}{4} x_{4,4} .
\end{aligned}
$$

For any molecular graph $G$ we have

$$
\begin{aligned}
& 2 x_{1,1}+x_{1,2}+x_{1,3}+x_{1,4}=n_{1}, \\
& x_{1,2}+2 x_{2,2}+x_{2,3}+x_{2,4}=2 n_{2}, \\
& x_{1,3}+x_{2,3}+2 x_{3,3}+x_{3,4}=3 n_{3}, \\
& x_{1,4}+x_{2,4}+x_{3,4}+2 x_{4,4}=4 n_{4} .
\end{aligned}
$$

Now we obtain $n_{2}, n_{3}$ and $n_{4}$ of the above system and since $n_{1}+n_{2}+n_{3}+n_{4}=n$, then 


$$
\frac{3}{2} x_{1,2}+\frac{4}{3} x_{1,3}+\frac{5}{4} x_{1,4}+x_{2,2}+\frac{5}{6} x_{2,3}+\frac{3}{4} x_{2,4}+\frac{2}{3} x_{3,3}+\frac{7}{12} x_{3,4}+\frac{1}{2} x_{4,4}=n .
$$

On the other hand

$$
x_{1,2}+x_{1,3}+x_{1,4}+x_{2,2}+x_{2,3}+x_{2,4}+x_{3,3}+x_{3,4}+x_{4,4}=m .
$$

By consider the two above equations and according to the results obtained in [16] we have

$$
\begin{aligned}
& x_{1,4}=\frac{4 n-2 m}{3}-\frac{4}{3} x_{1,2}-\frac{10}{9} x_{1,3}-\frac{2}{3} x_{2,2}-\frac{4}{9} x_{2,3}-\frac{1}{3} x_{2,4}-\frac{2}{9} x_{3,3}-\frac{1}{9} x_{3,4}, \\
& x_{4,4}=\frac{5 m-4 n}{3}+\frac{1}{3} x_{1,2}+\frac{1}{9} x_{1,3}-\frac{1}{3} x_{2,2}-\frac{5}{9} x_{2,3}-\frac{2}{3} x_{2,4}-\frac{7}{9} x_{3,3}-\frac{8}{9} x_{3,4} .
\end{aligned}
$$

By substituting these into the formula for $H(G)$, we have

$$
\begin{aligned}
H(G)=\sum_{1 \leq i \leq j \leq 4} \frac{2}{i+j} x_{i, j}= & \frac{3 m+4 n}{20}+\left(\frac{3}{4}-\frac{8}{15}\right) x_{1,2}+\left(\frac{19}{36}-\frac{20}{45}\right) x_{1,3}+\left(\frac{5}{12}-\frac{4}{15}\right) x_{2,2} \\
& +\left(\frac{10}{45}-\frac{5}{36}\right) x_{2,3}+\left(\frac{1}{6}-\frac{5}{12}\right) x_{2,4}+\left(\frac{5}{36}\right) x_{3,3}+\left(\frac{2}{7}-\frac{4}{15}\right) x_{3,4} .
\end{aligned}
$$

By calculate the coefficients of $x_{1,2}, x_{1,3}, x_{2,2}, x_{2,3}, x_{2,4}, x_{3,3}$ and $x_{3,4}$ we can see the all coefficients are positive and the minimum value of harmonic index for molecular graph with $n$ vertices and $m$ edges is $\frac{3 m+4 n}{20}$, that occur for graph are $x_{1,2}=x_{1,3}=x_{2,2}=x_{2,3}=x_{2,4}=x_{3,3}=x_{3,4}=0$, in other words, the molecular graph has only vertices of degree one and four. Also in [16] deduced

$$
\begin{aligned}
& x_{1,2}=2(n-m)-\frac{2}{3} x_{1,3}-\frac{1}{2} x_{1,4}+\frac{1}{3} x_{2,3}+\frac{1}{2} x_{2,4}+\frac{2}{3} x_{3,3}+\frac{5}{6} x_{3,4}+x_{4,4}, \\
& x_{2,2}=3 m-2 n-\frac{1}{3} x_{1,3}-\frac{1}{2} x_{1,4}-\frac{4}{3} x_{2,3}-\frac{3}{2} x_{2,4}-\frac{5}{3} x_{3,3}-\frac{11}{6} x_{3,4}-2 x_{4,4} .
\end{aligned}
$$

By substituting these into the formula for $H(G)$, we have

$$
\begin{aligned}
H(G)=\sum_{1 \leq i \leq j \leq 4} \frac{2}{i+j} x_{i, j}= & \frac{m+2 n}{6}+\left(\frac{1}{3}-\frac{4}{9}\right) x_{1,3}+\left(\frac{2}{5}-\frac{7}{17}\right) x_{1,4}+\left(\frac{2}{5}-\frac{4}{9}\right) x_{2,3} \\
& +\left(\frac{2}{3}-\frac{3}{4}\right) x_{2,4}+\left(\frac{7}{9}-\frac{5}{6}\right) x_{3,3}+\left(\frac{2}{7}-\frac{13}{36}\right) x_{3,4}+\left(\frac{11}{12}-1\right) x_{4,4} .
\end{aligned}
$$

By calculate the coefficients of $x_{1,3}, x_{1,4}, x_{2,3}, x_{2,4}, x_{3,3}, x_{3,4}$ and $x_{4,4}$ we can see the all coefficients are negative and the maximum value of harmonic index for molecular graph with $n$ vertices and $m$ edges is $\frac{m+2 n}{6}$, that occur for graph are $x_{1,3}=x_{1,4}=x_{2,3}=x_{2,4}=x_{3,3}=x_{3,4}=x_{4,4}=0$ in other words the molecular graph $G$ is either a path or a cycle.

Lemma 15: Among the connected molecular graphs having $n$ number of vertices considering two as the minimum degree for every vertex. For $n \geq 3$, the graph with only degrees two and without vertices of degree three and four is a molecular graph with the maximum harmonic index, which is equal to $\frac{n}{2}$. 
Proof: If $G$ be a molecular graph with $n$ vertices and $m$ edges with $\delta=2$, then $n \leq m \leq 2 n$. So, we have

$$
\begin{aligned}
& 2 x_{2,2}+x_{2,3}+x_{2,4}=2 n_{2}, \\
& x_{2,3}+2 x_{3,3}+x_{3,4}=3 n_{3}, \\
& x_{2,4}+x_{3,4}+2 x_{4,4}=4 n_{4}, \\
& n_{1}+n_{2}+n_{3}=n .
\end{aligned}
$$

By according the above system we can obtain

$$
x_{2,2}=n-\frac{5}{6} x_{2,3}-\frac{3}{4} x_{2,4}-\frac{2}{3} x_{3,3}-\frac{7}{12} x_{3,4}-\frac{1}{2} x_{4,4} \text {. }
$$

We can calculate the harmonic index of molecular graph with $\delta=2$, the following way

$$
H(G)=\sum_{2 \leq i \leq j \leq 4} \frac{2}{i+j} x_{i, j}=\frac{1}{2} x_{2,2}+\frac{2}{5} x_{2,3}+\frac{1}{3} x_{2,4}+\frac{1}{3} x_{3,3}+\frac{2}{7} x_{3,4}+\frac{1}{4} x_{4,4} .
$$

By substituting the $x_{2,2}$ into the formula for $H(G)$, we have

$$
H(G)=\sum_{2 \leq i \leq j \leq 4} \frac{2}{i+j} x_{i, j}=\frac{n}{2}+\left(\frac{2}{5}-\frac{5}{12}\right) x_{2,3}+\left(\frac{1}{3}-\frac{3}{8}\right) x_{2,4}+\left(\frac{2}{7}-\frac{7}{24}\right) x_{3,4} .
$$

By calculate the coefficients of $x_{2,3}, x_{2,4}$ and $x_{3,4}$ we can see the all coefficients are negative and the maximum value of harmonic index for molecular graph with $\mathrm{n}$ vertices and $\mathrm{m}$ edges with $\delta=2$ is $\frac{n}{2}$, that occur for graph are $x_{2,3}=x_{2,4}=x_{3,3}=x_{3,4}=x_{4,4}=0$, in other words the molecular graph $G$ is a cycle.

Lemma16: Let $U$ be the unicyclic molecular connected graph of order $n$ with $p$ pendent vertices. Then

$$
H(U) \geq \frac{n}{4}+\frac{3 p}{20} .
$$

With the equality holding if and only if all pendent vertices are adjacent to the vertices of degrees are 4 and $x_{i, j}=0$ for $1 \leq i \leq j \leq 4$ and $(i, j) \neq(1,4),(4,4)$.

Proof: We consider the notations on Section 2, so we can write

$$
\begin{aligned}
H(G)= & \frac{2}{3} x_{1,2}+\frac{1}{2} x_{1,3}+\frac{2}{5} x_{1,4}+\frac{1}{2} x_{2,2}+\frac{2}{5} x_{2,3}+\frac{1}{3} x_{2,4}+\frac{1}{3} x_{3,3}+\frac{2}{7} x_{3,4}+\frac{1}{4} x_{4,4} \\
= & \frac{2}{3} x_{1,2}+\frac{1}{2} x_{1,3}+\frac{2}{5}\left(p-x_{1,2}-x_{1,3}\right)+\frac{1}{2} x_{2,2}+\frac{2}{5} x_{2,3}+\frac{1}{3} x_{2,4}+\frac{1}{3} x_{3,3} \\
& +\frac{2}{7} x_{3,4}+\frac{1}{4}\left(n-p-x_{2,2}-x_{2,3}-x_{2,4}-x_{3,3}-x_{3,4}\right) \\
= & \frac{n}{4}+\frac{3 p}{20}+\left(\frac{2}{3}-\frac{2}{5}\right) x_{1,2}+\left(\frac{1}{2}-\frac{2}{5}\right) x_{1,3}+\left(\frac{1}{2}-\frac{1}{4}\right) x_{2,2} \\
& +\left(\frac{2}{5}-\frac{1}{4}\right) x_{2,3}+\left(\frac{1}{3}-\frac{1}{4}\right) x_{2,4}+\left(\frac{1}{3}-\frac{1}{4}\right) x_{3,3}+\left(\frac{2}{7}-\frac{1}{4}\right) x_{3,4} .
\end{aligned}
$$


By calculate the coefficients of $x_{1,2}, x_{1,3}, x_{2,2}, x_{2,3}, x_{2,4}, x_{3,3}$ and $x_{3,4}$ we can see the all coefficients are positive and the minimum value of harmonic index for unicyclic molecular graph with $n$ vertices and $n$ edges with $p$ pendent vertices is $\frac{n}{4}+\frac{3 p}{20}$, that occur for unicyclic molecular graph are $x_{1,2}=x_{1,3}=x_{2,2}=x_{2,3}=x_{2,4}=x_{3,3}=x_{3,4}=0$, in other words, the unicyclic molecular graph has only vertices of degree one and four.

Theorem 17: Let $G$ be a connected graph of order $n$ and maximum degree $\Delta$, where $2 \leq \Delta \leq n-1$. Then

$$
H(G) \geq \frac{2 n \Delta}{(1+\Delta)^{2}} .
$$

With the equality if and only if $G \cong S_{n}$.

Proof: Since $G$ is a graph of order $\mathrm{n}$ and maximum degree $\Delta$, we have

$$
\begin{aligned}
& 2 x_{1,1}+x_{1,2}+\cdots+x_{1, \Delta}=n_{1}, \\
& x_{1,2}+2 x_{2,2}+\cdots+x_{2, \Delta}=2 n_{2}, \\
& \vdots \\
& x_{1, \Delta}+x_{2, \Delta}+\cdots+2 x_{\Delta, \Delta}=\Delta n_{\Delta} .
\end{aligned}
$$

And

$$
n_{1}+n_{2}+\cdots+n_{\Delta}=n .
$$

Now we can calculate the harmonic index as following that

$$
H(G)=\sum_{1 \leq i \leq j \leq \Delta} \frac{2}{i+j} x_{i, j} .
$$

According to the first and last equations of (49) and equation (50) we have,

$$
\begin{aligned}
x_{1, \Delta} & =\frac{\Delta}{1+\Delta}\left(n-\sum_{2 \leq j \leq \Delta-1}\left(1+\frac{1}{j}\right) x_{1, j}-\sum_{2 \leq i \leq \Delta-1} \frac{1}{i} x_{i, \Delta}-\sum_{2 \leq i \leq j \leq \Delta-1}\left(\frac{1}{i}+\frac{1}{j}\right) x_{i, j}\right) \\
& -\frac{1}{1+\Delta}\left(\sum_{2 \leq i \leq \Delta-1} x_{i, \Delta}+2 x_{\Delta, \Delta}\right) .
\end{aligned}
$$

By substituting the $x_{1, \Delta}$ into the formula for $H(G)$, we have

$$
H(G)=\frac{2 n \Delta}{(1+\Delta)^{2}}+\sum_{1 \leq i \leq j \leq \Delta,(i, j) \neq(1, \Delta)} f(i, j, \Delta) x_{i, j} .
$$

Where $f(i, j, \Delta)=\frac{2}{i+j}-\frac{2 \Delta}{(1+\Delta)^{2}}\left(\frac{1}{i}+\frac{1}{j}\right)$, and we will prove that $f(i, j, \Delta)$ is nonnegative for $1 \leq i \leq j \leq \Delta,(i, j) \neq(1, \Delta)$. On the other hand,

$$
\frac{2}{i+j}-\frac{2 \Delta}{(1+\Delta)^{2}}\left(\frac{1}{i}+\frac{1}{j}\right) \geq 0 \Leftrightarrow(j \Delta-i)(i \Delta-j) \geq 0 \text {. }
$$


The above non-equation is true, because $1 \leq i \leq j \leq \Delta$. Then the minimum value of the harmonic index with the maximum degree $\Delta$ is $\frac{2 n \Delta}{(1+\Delta)^{2}}$ that the equality accrues for graph with $x_{1, \Delta}=\frac{n \Delta}{1+\Delta}$ and $x_{i, j}=0$ for $1 \leq i \leq j \leq \Delta,(i, j) \neq(1, \Delta)$. It is clear that for $\Delta=n-1$ the $x_{1, \Delta}$ is integer. On the other hand, $G \cong S_{n}$.

Corollary 18: Let $f(x, y)=\frac{2}{x+y}$, where $x, y \geq 1$. If $y \geq 1$ is fixed, then $f(x, y)$ is decreasing for $x$.

Corollary 19: Let $G$ be a simple connected graph with $n$ vertices, $m$ edges, maximum vertex degree $\Delta$ and $p$ pendent vertices. Then

$$
H(G) \geq \frac{2 p}{1+\Delta}+\frac{m-p}{\Delta} .
$$

The equality holds if and only if $G \in \Gamma$.

Proof: Since $G$ is a simple connected graph with $n$ vertices, $m$ edges and maximum degree $\Delta$, then we can calculate the harmonic index by

$$
H(G)=\sum_{1 \leq i \leq j \leq \Delta} \frac{2}{i+j} x_{i, j} .
$$

Now by corollary 18 , we have

$$
\begin{aligned}
H(G) & =\sum_{2 \leq j \leq \Delta} \frac{2}{1+j} x_{1, j}+\sum_{2 \leq i \leq j \leq \Delta} \frac{2}{i+j} x_{i, j} \\
& \geq\left(\sum_{2 \leq j \leq \Delta} x_{1, j}\right) \frac{2}{1+\Delta}+\left(\sum_{2 \leq i \leq j \leq \Delta} x_{i, j}\right) \frac{1}{\Delta}=\frac{2 p}{1+\Delta}+\frac{m-p}{\Delta} .
\end{aligned}
$$

Now we consider the equality. The equality holding if and only if $x_{1, j}=0(2 \leq j \leq \Delta)$ and $x_{i, j}=0(2 \leq i \leq j \leq \Delta$ and $(i, j) \neq(\Delta, \Delta))$. It is clear that $G \in \Gamma$.

\section{Numerical example}

In [9] the authors have shown correlation between the geometric-arithmetic index and some phyisico-chemical properties. In this section we calculate the harmonic, geometric-arithmetic and sum connectivity indices for octane isomers (see Table 1). We obtain the correlation between harmonic index and geometric-arithmetic and sum connectivity indices. The linear correlations between $H$ and both $G A_{1}$ and $\chi$ are given below

$$
H(G)=1.077( \pm 0.021) G A_{1}(G)-3.598( \pm 0.138), \quad R=0.997 \text {, }
$$

and 
$H(G)=1.933( \pm 0.031) \chi(G)-3.226( \pm 0.106), \quad R=0.998$.

\begin{tabular}{|l|l|c|c|c|}
\hline$\#$ & Octane's & $H$ & $G A_{1}$ & $\chi$ \\
\hline 1 & n-Octane & 3.83333 & 6.88562 & 3.65470 \\
\hline 2 & 2-Metherlheptane & 3.56667 & 6.65466 & 3.52456 \\
\hline 3 & 3-Metherlheptane & 3.63333 & 6.71124 & 3.54913 \\
\hline 4 & 4-Metherlheptane & 3.63333 & 6.71124 & 3.54913 \\
\hline 5 & 2,2-dimethyl-hexane & 3.20000 & 6.28562 & 3.32724 \\
\hline 6 & 3,3-dimethyl-hexane & 3.30000 & 6.37124 & 3.36562 \\
\hline 7 & 2,3-dimethyl-hexane & 3.40000 & 6.52068 & 3.43281 \\
\hline 8 & 2,3-dimethyl-hexane & 3.36667 & 6.48027 & 3.41899 \\
\hline 9 & 2,5-dimethyl-hexane & 3.30000 & 6.42369 & 3.39443 \\
\hline 10 & 3,4-dimethyl-hexane & 3.46667 & 6.57726 & 3.45738 \\
\hline 11 & 2,3,4-trimethyl-pentane & 3.16667 & 6.33013 & 3.31650 \\
\hline 12 & 2,2,3-trimethyl-pentane & 3.05238 & 6.17837 & 3.24417 \\
\hline 13 & 2,2,4-trimethyl-pentane & 2.93333 & 6.05466 & 3.19710 \\
\hline 14 & 2,3,3-trimethyl-pentane & 3.08571 & 6.20741 & 3.25799 \\
\hline 15 & 2,2,3,3-tetramethyl-butane & 2.65000 & 5.80000 & 3.03683 \\
\hline 16 & 2-methyl-3-ethyl-pentane & 3.46667 & 6.57726 & 3.45738 \\
\hline 17 & 3-methyl-3-ethyl-pentane & 3.40000 & 6.45685 & 3.40401 \\
\hline 18 & 3-Ethyl-hexane & 3.70000 & 6.76781 & 3.57369 \\
\hline
\end{tabular}

Table 1: The $H, G A_{1}$ and $\chi$ indices of the octane isomers.

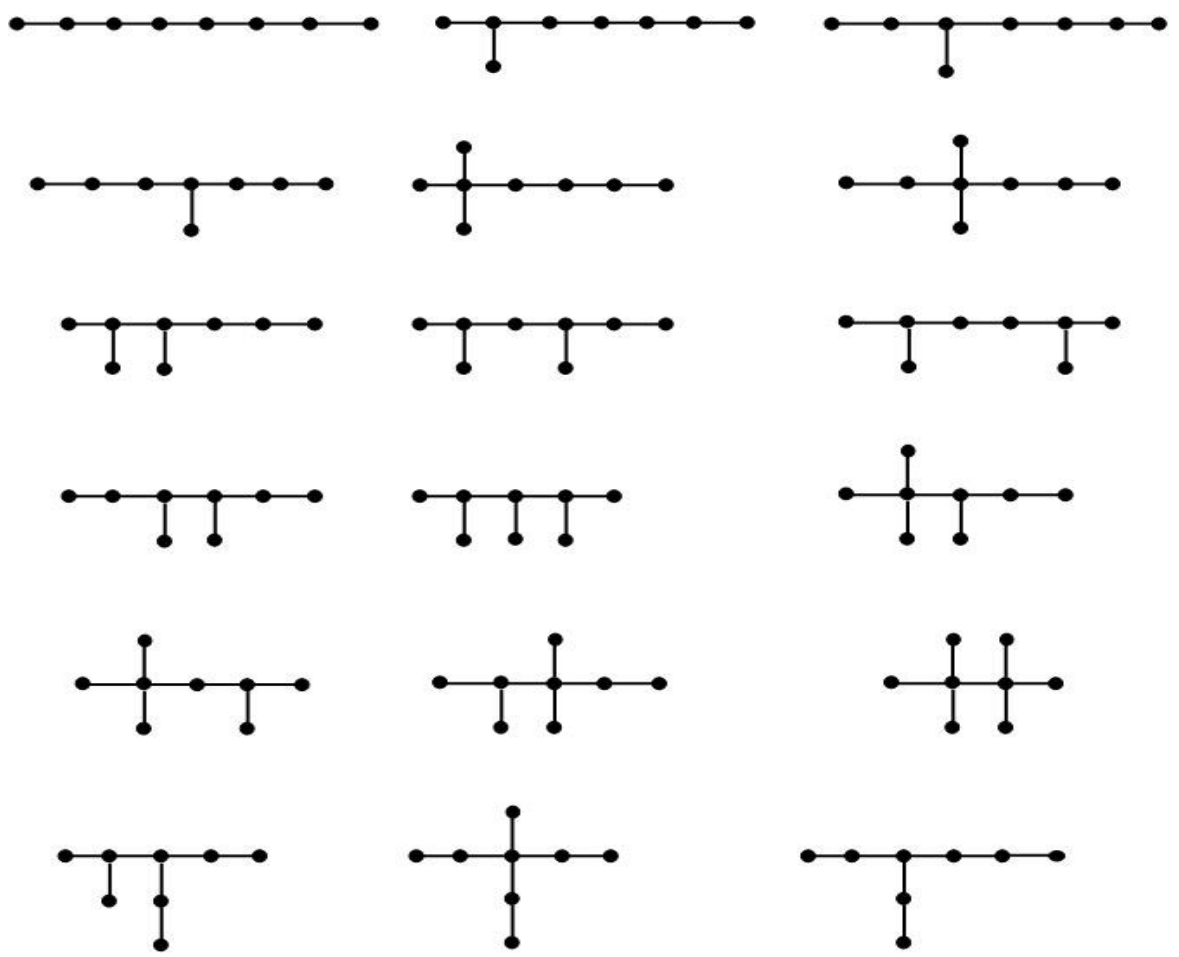

Figure 1: All octane isomers 

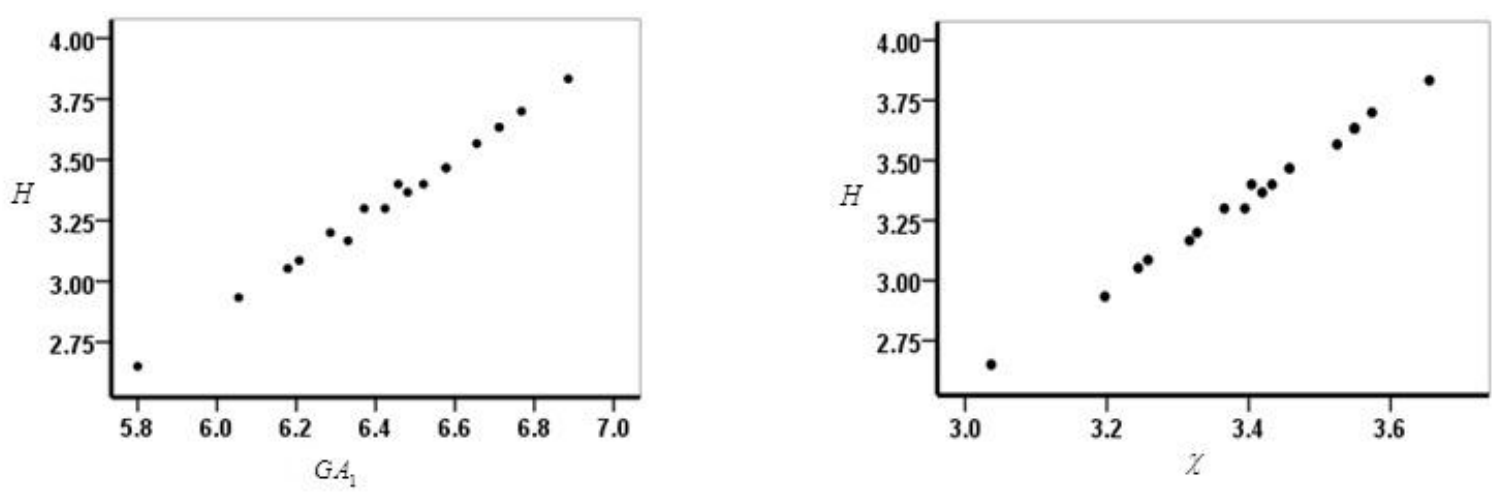

Figure 2: Graphs showing correlation between $H, G A_{1}$ and $H, \chi$ index respectively.

\section{Conclusion}

In this report we obtain the relation between harmonic index and another topological index. According to obtain results in relation between harmonic index and sum-connectivity index but this problem that for what kind of graphs we have $H(G) \leq \chi(G)$ is an open problem.

\section{References}

[1] S. Fajtlowicz, on conjectures of Graffiti-II, Congr. Numer. 60 (1987) 187-197.

[2] O. Favaron, M. Maho, J. F. Sacl, Some eigenvalue properties in graphs (conjectures of Graffiti-II), Discrete Math., 111 (1993) 197-220.

[3] L. Zhong, The harmonic index for graphs, Appl. Math. Let. 25 (2012) 561-566.

[4] R. Wua, Z. Tanga, H. Deng, A lower bound for the harmonic index of a graph with minimum degree at least two, Published by Faculty of Sciences and Mathematics, University of Nis, Serbia 27 (2013) 51-55.

[5] X. Xu, Relationships between harmonic index and other topological indices, App. Math. Sciences 6 (2012) 2013 - 2018.

[6] H. Deng, S. Balachandran, S. K. Ayyaswamy, Y. B. Venkatakrishnan, on the harmonic index and thechromatic number of a graph, Discrete Appl. Math., 161 (2013) 2740-2744.

[7] L. HongboHua, The Harmonic index of general graphs, nanocones and triangular benzenoid graphs, Optoelectronics Adv. Materials Communi., 6 (2012) 660 - 663.

[8] R. Chang, Y. Zhu, on the harmonic index and the minimum degree of a graph, Rom. J. Information Scie. Tech., 15 (2012) 335- 343.

[9] D. Vukicevic, B. Furtula, Topological index based on the ratios of geometrical and arithmetical means of end-vertex degrees of edges, J. Math. Chem., 46 (2009) 1369-1376. 
[10] Y. Yuan, B. Zhou, N. Trinajstic, on geometric-arithmetic index, J. Math. Chem., 47 (2010) 833-841.

[11] B. Zhou, N. Trinajstic, on a novel connectivity index, J. Math. Chem. 46 (2009) 1252-1270.

[12] Z. Du, B. Zhou, N. Trinajstic, Minimum sum-connectivity indices of trees and unicyclic graphs of a given matching number, J. Math. Chem., 47 (2010) 842-855.

[13] R. Xing, B. Zhou, N. Trinajstic, Sum-connectivity index of molecular trees, J. Math. Chem., 48 (2010)583-591.

[14] J. A. Bondy, U. S. R. Murty, Graph theory with application, New York: North Holland, 1976.

[15] N. Trinajstic, Chemical graph theory, 2nd revised ed (CRC Press, Boca Raton, FL, 1992).

[16] I. Gutman, O. Miljkovic, Molecules with smallest connectivity indices, Match Commun. Math. Comput. Chem., 41 (2000) 57-70. 